Cataclysmic Variables, Symbiotic Stars, and Related Binaries 


\title{
MWC 560: ITS SPECTROSCOPIC AND PHOTOMETRIC DEVELOPMENT IN 1990
}

\author{
D.A.H. BUCKLEY \\ South African Astronomical Observatory \\ P.O. Box 9 \\ Observatory 7935 , \\ Cape Town \\ South Africa
}

\begin{abstract}
Optical spectroscopic, photometric, and infrared photometric observations of the symbiotic-like star MWC560, which underwent a prolonged 'outburst' during 1990, are presented. Both the magnitudes and timescales of outbursts are similar to other symbiotics, and related systems. The large ejection velocities observed, and the transient nature of the highly blue shifted absorption components, are quite atypical, however.
\end{abstract}

\section{Introduction}

In this brief report we present observations of MWC560 during the recently reported [1] 1990 outburst, obtained mainly in the period March-May 1990 at the South African Astronomical Observatory (SAAO).

MWC560 exhibited similar characteristics to those reported for the 1984 outburst [2], specifically the highly transient, and extremely blue-shifted (up to $\sim-5000 \mathrm{~km} / \mathrm{s}$ ) Balmer absorption components $[2,3,4]$. Likewise photometric 'flickering', with a timescale of order 15-20 minutes, was evident $[2,4,5]$.

An initial model [1] interpreted the optical spectra, and in particular the variable, highly blue shifted $\mathrm{H} \beta$ absorption components, as arising from a collimated jet of outflowing material. Alternatively, some recently reported IUE and optical observations have suggested ejection of an optically thick shell at high velocities [6].

\section{Spectroscopy}

All observations were conducted using the Reticon Photon Counting System (RPCS) detector on the Cassegrain spectrograph of the SAAO $1.9 \mathrm{~m}$ telescope. Since most of the observations were obtained through cooperation with scheduled observers, the spectra cover different wavelength regions at a variety of resolutions (from $\sim 1$ to $3 \AA$ ). In Figure 1 we present a sample of the 'blue' spectra in the

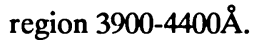

Immediately obvious are the transient blue-shifted Balmer components, which can completely disappear in an interval of $\sim 1$ day (e.g. see 9/10 March and 22/23 March). Other features include the redward Balmer emission components, and in the higher resolution data (e.g. 9-1.1 March), weak

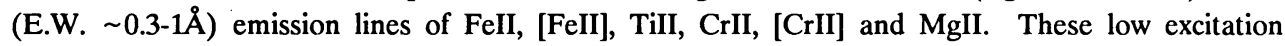


multiplets are also seen in other symbiotic systems, and are thought to arise in the red giant's atmosphere.

These spectra, and those already published [1], are quite clearly different to the discovery spectrum, and even to a more recent digital spectrum [7,8], both of which are described as M4e. Close inspection of our spectra in the region $\mathrm{H} \alpha$ reveal the veiled TiO bandhead at $6159 \AA$, albeit weakly, which establishes the presence of the late-type companion.

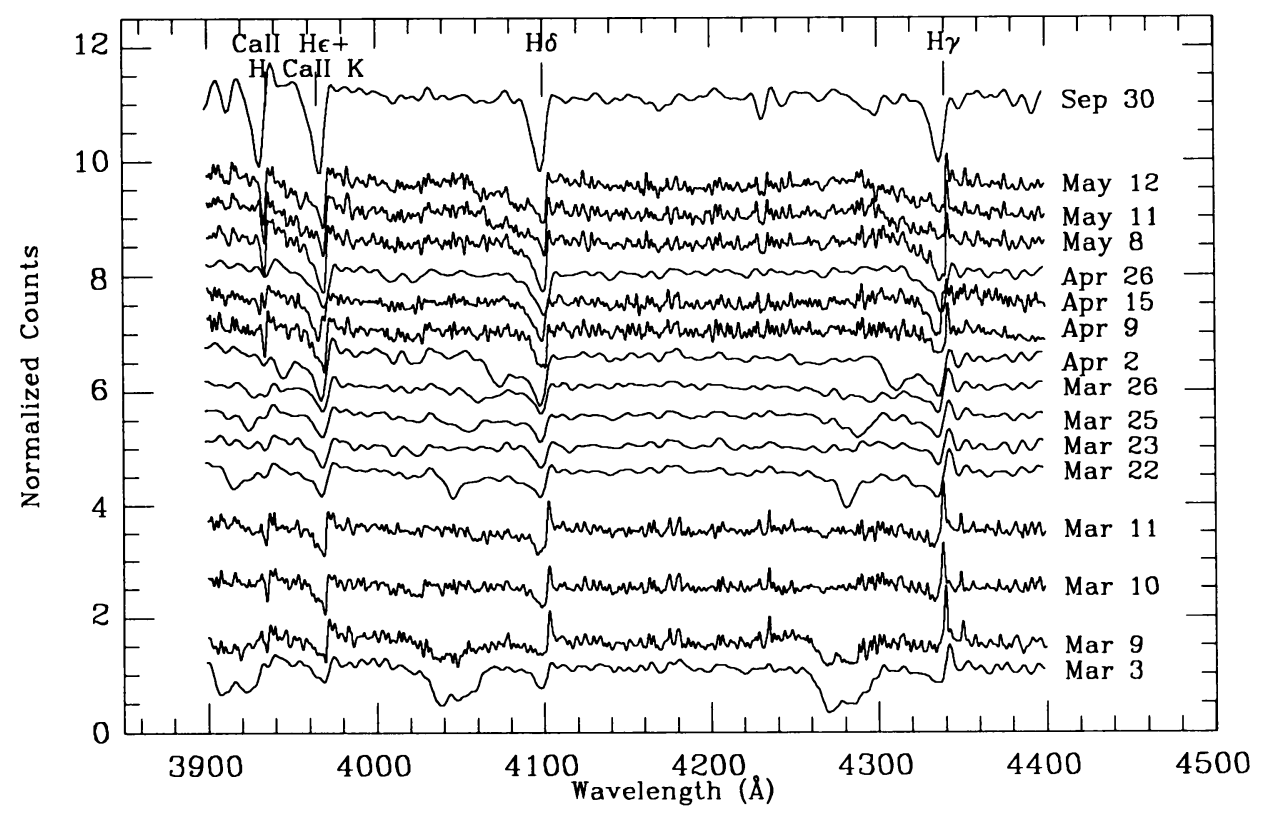

Figure 1. Blue spectra of MWC560 taken during 1990 at SAAO

\section{Velocities and Line Variations}

In some instances up to 4 absorption components of the Balmer lines were found, which showed marked variations in strength, and position, on relatively short timescales (days). Whereas the earlier spectra could often be easily resolved into various high velocity components, the spectra taken in the April-May period showed more 'diffuse', or highly asymmetric, blue shifted components. These distinct P Cygni profiles still extend some $3000 \mathrm{~km} / \mathrm{s}$ bluewards. Less chaotic in behaviour are the 'mildly' blue shifted absorption lines, declining from $-500 \mathrm{~km} / \mathrm{s}$ (March) to $-200 \mathrm{~km} / \mathrm{s}$ (May). The equivalent widths of these least blue shifted components also appear to be relatively stable. No correlation exists between radial velocity and line FWHM for any of the absorption components.

Balmer emission line velocities were constant at $\sim 100 \mathrm{~km} / \mathrm{s}$, while the weak FeII \& [FeII] emission lines were mostly somewhat lower $(+60$ to $80 \mathrm{~km} / \mathrm{s})$. Finally, the large Balmer decrement possibly increases with time, though varying spectral resolution and data quality preclude a definitive statement on this point. 


\section{Optical Photometry}

$\mathrm{UBV}(\mathrm{RI})_{c}$ and limited high-speed (2 sec resolution) U-band photometry of MWC560 was conducted on the SAAO $0.5 \mathrm{~m}$ telescope in the period from 28 February to 12 May 1990. Magnitudes and colours of MWC560 are presented in Figure 2, where we have also included published V \& UBV photometry $[1,5]$ as a comparison. Colour variations as a function of $\mathrm{V}$ indicate that a fairly strong positive correlation exists between $\mathrm{V}$ and the V-R and V-I colour indices, but not with U-B or B-V. This is likely due to the presence of the strong transient absorption features, which are most pronounced in the blue part of the spectrum. Analysis of several high-speed optical photometry runs failed to reveal any coherent periodicities up to the Nyquist frequency.
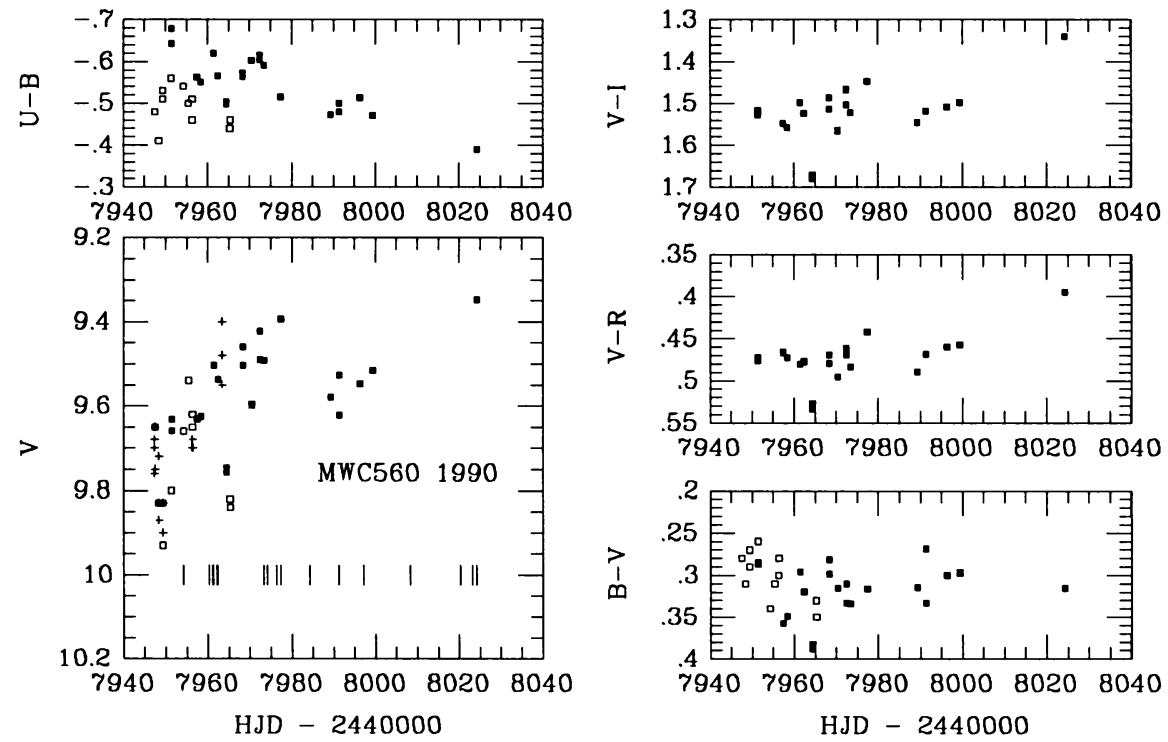

Figure 2. Optical (UBVRI) light \& colour curves of MWC560. Filled squares: SAAO data, open squares: data from [1], crosses: data from [6]. Vertical bars indicate when spectra were obtained at SAAO.

\section{Infrared Photometry}

Infrared (JHKL) photometry of MWC560 was first undertaken at SAAO from 1984 to early 1986, and further observations were obtained during the 1990 outburst. The IR colours in both the 1984-1986 and 1990 seasons are basically the same, with the small change possibly due to the M-star being slightly hotter, or somewhat less reddened. The IR colours are typical of low amplitude $M$ giants, and actually similar to $M$ stars found in recurrent novae [9]. Both the IR colours and the aforementioned presence of TiO bands in the red spectra unequivocally establish the presence of an M4 star in the system. 


\section{Discussion}

The most remarkable aspect of MWC560 is the large ejection velocities observed, and the transient nature of the highly blue shifted absorption lines. Such large velocities are only usually typical of very fast novae. The A to F spectral type, which seems to characterize MWC560 during outburst, is reminiscent of spectra of novae, recurrent novae, some symbiotic novae (also termed very slow novae), and even normal outbursting symbiotic stars, all at maximum light [e.g. 10,11]. The correlation of the $\mathrm{V}$ with the indices (V-R \& V-I) demonstrates that MWC560 was redder when it was fainter, as is observed in symbiotic outbursts [11].

A recently published archival photographic light curve [12] shows that MWC560 only brightens by $\sim 3$ mag during its outbursts, and that historically there have probably been 5-6 such events since 1928 . Such a low amplitude is in keeping with the apparently slow spectral evolution during 1990 . The timescales of the previous outbursts [12] would seem to indicate closer similarities with normal outbursting symbiotics, though of the low excitation variety (e.g. TX CVn).

Both the optical and UV absorption lines show similarities in their P Cyg structure to other symbiotic-like systems, for instance the presumed symbiotic nova PU Vul [14], and the unusual Be star XX Oph [6,13]. However an important caveat must be added, namely that the velocities are at least an order of magnitude higher!

The low degree of optical brightening during outburst suggests that the ejection is either highly non-symmetric (i.e. like in a jet), or that any ejected shell is extremely fragmented, with a low integrated surface area.

The future spectral and photometric evolution, and possible radio or X-ray detections, will hopefully help to illucidate the nature of this interesting system.

\section{ACKNOWLEDGEMENTS}

The following people contributed to the observations of MWC560: L. Balona, B. Carter, T. Lloyd Evans, A.P. Fairall, M. Feast, D. Holmgren, C.D. Laney, F. Marang, G. Roberts, W. Slawson, P.A. Whitelock, H. Winkler, F. van Wyk and A. Zijlstra. Useful discussions with P.A. Whitelock are gratefully acknowledged.

\section{REFERENCES}

1. Tomov, T. et al. (1990), Nature, 346, 637.

2. Bond, H.W. et al. (1984), Bull. Am. Astr. Soc., 16, 516.

3. Maran, S.P., Michalitsianos, A.G. \& Oliversen, R.J. (1990), LAUC 4979.

4. Buckley, D.A.H. et al. (1990), LAUC 4980.

5. Dapergolas, A., Kontizas, E. \& Kontizas, M. (1990), LAUC 4982.

6. Michalitsianos, A.G. et al. (1991), Ap. J., 371, 761.

7. Merrill, P.W. \& Burwell, C.G. (1943), Ap.J., 98, 153.

8. Allen, D.A. (1978), M.N.R.A.S., 184, 601.

9. Sekiguchi, K. et al. (1990), M.N.R.A.S., 246, 78.

10. Warner, B. (1989), 'Properties of Novae: An Overview', in Classical Novae, ed. M.F. Bode \& A Evans, Wiley, Chichester.

11. Kenyon, S. (1989), The Symbiotic Stars, Cambridge University Press, Cambridge.

12. Luthardt, R. (1991), IBVS No. 3563.

13. Maran, S.P. et al. (1991), Nature, 350, 404.

14. Iijima, T. and Ortolani, S. (1984), Astr. Ap., 136, 1. 